\title{
High-Prevalence of Various High-Risk Sub-types of Human Papilloma Virus in Patients with Acquired Cholesteatoma in Greece
}

\section{Anargyros Skoulakis ${ }^{1}$, Vasileios Lachanas ${ }^{2}$, Zoe Florou ${ }^{1}$, Maria Samara ${ }^{3}$, Myrto Mantzana-Petinelli ${ }^{1}$, Efthymia Petinaki $^{1}$ and Charalampos Skoulakis ${ }^{2 *}$}

${ }^{1}$ Department of Microbiology, School of Medicine, University of Thessaly, Larissa, Greece

${ }^{2}$ Department of Otorhinolaryngology, University Hospital of Larissa, Greece

${ }^{3}$ Department of Pathology, School of Medicine, University of Thessaly, Greece

*Corresponding Author: Charalampos Skoulakis, Associate Professor, Department of Otorhinolaryngology, University Hospital of Larissa, Greece.

Received: February 09, 2018; Published: March 03, 2018

DOI: 10.31080/ASMI.2018.01.0031

\begin{abstract}
Although there are a lot of theories, the pathogenesis of cholesteatoma remains unclear. Few studies exist worldwide regarding the presence of Human Papilloma Virus (HPV) in cholesteatoma with controversial results. The presence of DNA of various high and low risk HPV sub-types was detected by real-time PCR to lesions obtained from sixty-two patients with acquired cholesteatoma. In addition, the presence of bacterial DNA was also detected using $16 \mathrm{~S}$ rRNA PCR. Control group included tympanic membrane skin samples from patients operated by tympanoplasty. Cholesteatoma samples of thirty patients (48.3\%) were found to be positive for one at least HPV sub-type. Twenty-five samples were positive for HPV16 (40.3\%), nine were positive for HPV6 (14.5\%), one for HPV18 (1.6\%), one for HPV31 (1.6\%), one for HPV51 (1.6\%) and one for HPV59 (1.6\%). None of the patients of control group was found to be positive for any sub-type HPV (0\%). On the other hand, all specimens (cholesteatoma and tympanic membrane skin mucosa) were negative for the presence of bacterial DNA. Our results demonstrate a high prevalence of various high and low-risk HPV sub-types in cholesteatoma tissue samples in Central Greece.
\end{abstract}

Keywords: HPV; sub-types acquired holesteatoma; Greece

\section{Introduction}

Cholesteatoma is an abnormal, noncancerous skin growth tumor, that can develop in the middle section of the ear, behind the eardrum [1]. It may be a birth defect (congenital cholesteatoma) or acquired cholesteatoma, which is most commonly associated with repeated middle ear chronic inflammation caused by bacteria or viruses $[2,3]$.

Papillomaviruses are small, non-enveloped, epitheliotropic, double stranded DNA viruses that infect mucosal and cutaneous epithelia in a wide variety of higher vertebrates in a species-specific manner and induce cellular proliferation [4,5]. It is well known that Human Papilloma Virus (HPV) infection has been mainly associated with cervical cancer but also with anal and oropharyngeal cancer [6,7]. In 1995, Stremlau., et al. reported for the first time the presence of HPV DNA [8]; however, up to date, it remains unclear whether the HPV is a possible etiology for the development of cholesteatoma.

\section{Aim of the Study}

Aim of the present study was to determine the prevalence and the distribution of various HPV sub-types in patients with acquired cholesteatoma in Central Greece.

\section{Materials and Methods}

This study was conducted prospectively on tissues collected from patients who were operated with acquired cholesteatoma from January 2008 to January 2017 in the Department of Otorhinolaryngology of the University Hospital of Larissa (UHL), Central Greece. Patients with congenital cholesteatoma were excluded. We note that UHL is a tertiary care unit that serves a population of 1.000.000 inhabitants.

For the purpose of the present study, fresh tissues from patients who were operated with acquired cholesteatoma were sent to the Department of Microbiology of UHL. Extraction of total DNA was assessed using commercial kits (Invitrogen), according to the manufacturer's instructions. The efficiency of DNA extraction and the possible presence of inhibitors in the sample were confirmed by the detection of $\beta 2$-globin gene [9]. The extracted DNA samples were tested by quantitative real-time PCR (Applied Biosystems 7500 Fast Real-Time PCR system) according to the manufacturer's instructions, using two commercially available assays, the HPV High Risk Screen Real Time PCR (Sacace Biotechnologies), which detects the sub-types $16,18,31,33,35,39,45,51,52,56,58,59$, 66, 68 and the HPV 6/11 Real-Time PCR kit (Sacace Biotechnologies), which detects the sub-types 6 and 11. In addition, the pres- 
ence of bacterial DNA was also tested in all specimens using $16 \mathrm{~S}$ broad range PCR followed by sequencing [10]. In addition, sixty clinical samples included tympanic membrane skin, which were obtained from patients who were operated by tympanoplasty the same period, were used as control group; DNA extraction followed by molecular detection of HPV and bacteria was performed as previously described. We note that, as the origin of cholesteatoma is the tympanic membrane skin, we chose patients who were operated by tympanoplasty as control group.

All calculations were computed by R-base program for Linux. The results were presented as mean $\bigotimes$ SD or percentages. SPSS 20 statistical software (IBM, Chicago, IL, USA) was used for statistical analysis. P values lower than 0.05 were considered as statistically significant.

The study was approved by the University Hospital of Larissa and School of Medicine, University of Thessaly review boards (approval protocol No 19/2-2-2017). A written informed consent within demographic information of all participants (age, gender, history etc) was obtained.

\section{Results}

Our study group consisted of sixty-two patients (38 men and 24 women) aged from 19-77 years old (mean age $40.1 \otimes 20.4$ ), while, the control group included sixty patients (18 men and 24 women), aged from $30-55$ years old (mean age $42 \otimes 4$ ). DNA extraction, indicated by $\beta 2$-globin gene detection, was successfully done in all samples. Cholesteatoma samples of 30 patients (48.3\%) were found to be positive for one at least HPV subtype. Twenty-five samples were positive for HPV16 (40.3\%), nine were positive for HPV6 (14.5\%), one for HPV18 (1.6\%), one for HPV31 (1.6\%), one for HPV51 (1.6\%) and one for HPV59 (1.6\%). It is interesting that, among the HPV-positive samples, six were positive for both HPV16 and HPV6, while, one was positive for HPV6, HPV16 and HPV18. None of the patients of control group was found to be positive for any HPV sub-type $(0 \%)$. Statistical significant difference on the prevalence of HPV was observed between the two study groups ( $\mathrm{p}$ $<0.005)$.

Finally, all specimens (cholesteatoma and tympanic membrane skin) were negative for the presence of bacterial DNA, indicating that bacteria don't have essential role in the pathogenesis of cholesteatoma.

\section{Discussion}

In our study, a high prevalence of various high-risk HPV subtypes was detected in tissues collected from patients with acquired cholesteatoma, while, none specimen of the control group was found to be positive. As mentioned above, the HPV prevalence in cholesteatomas varies significantly between studies. Rydzewski., et al. have demonstrated that, among a group of fifty-three patients, of whom thirty-nine suffered from granulation tissue chronic otitis media, seven from cholesteatomatous otitis media, six from middle ear malignant neoplasm, and one from middle and/or external ear benign neoplasm, the presence of HPV DNA reached to 41.5\% [11]; specifically, among cholesteatomatous chronic otitis media HPV DNA types 6 or 11 was identified in 70\%. On the other hand, Bai., et al. reported 29,5\%, prevalence of HPV DNA in forty-four cases of middle ear cholesteatoma, while Bergmann., et al. reported $36 \%$ of cholesteatomas to contain HPV DNA using PCR [12,13].

In addition, Ferekidis., et al. demonstrated that among fourteen specimens (seven aggressive and seven simple cholesteatomas), three aggressive were HPV-positive, while, all simple cholesteatomas were HPV negative [14]. On the contrary, Chao., et al. reported that only one out of thirty-six cholesteatomas $(2,7 \%)$ contained 6-HPV DNA, while, Franz., et al. showed that only one out of twenty-nine $(3,4 \%)$ biopsies of cholesteatomas was positive for HPV DNA $[15,16]$.

\section{Conclusion}

Our results demonstrate a high prevalence of various HPV subtypes (48.3\%) in patients with acquired cholesteatoma in Central Greece, suggesting that the virus might play a role in the pathogenesis of cholesteatomas. Other studies are required to clarify if the viral presence influences the progression of cholesteatoma and if the virus is responsible for recurrences.

\section{Bibliography}

1. Jennings BA., et al. "The genetics of cholesteatoma. A systematic review using narrative synthesis". Clinical Otolaryngology 43.1 (2018): 55-67.

2. Nguyen KH., et al. "Possible participation of acidic $\mathrm{pH}$ in bone resorption in middle ear cholesteatoma". Laryngoscope 124.1 (2014): 245-250.

3. Hamed MA., et al. "Pathogenesis and Bone Resorption in Acquired Cholesteatoma: Current Knowledge and Future Prospectives". Clinical and Experimental Otorhinolaryngology 9.4 (2016): 298-308.

4. Milner DA. "Diagnostic Pathology: Infectious Diseases". $1^{\text {st }}$ edition. Elsevier Health Sciences (2015): 40.

5. Schiller JT., et al. "Current understanding of the mechanism of HPV infection". Gynecologic Oncology 118.1 (2010): S12S17.

6. Gao G, et al. "Human Papillomavirus and the Development of Different Cancers". Cytogenetic and Genome Research 150.3-4 (2016): 185-193.

7. Westra WH. "The changing face of head and neck cancer in the $21^{\text {st }}$ century: the impact of HPV on the epidemiology and pathology of oral cancer". Head and Neck Pathology 3.1 (2009): 78-81. 
8. Stremlau A., et al. "Detection of DNA of human papillomaviruses (HPV) in an "aggressively" growing cholesteatoma. Is cholesteatoma a virus-induced tumor?" HNO 43.1 (1995): 3-5.

9. Ioannidis D., et al. "Herpes viruses and human papilloma virus in nasal polyposis and controls". Brazilian Journal of Otorhinolaryngology 81.6 (2015) : 658-662.

10. Gatselis N., et al. "Direct detection of Cardiobacterium hominis in serum from a patient with infective endocarditis by broad-range bacterial PCR". Journal of Clinical Microbiology 44.2 (2006): 669-672.

11. Rydzewski B., et al. "Identification of human papilloma viruses (HPV) in inflammatory states and ear neoplasms". Otolaryngologia Polska 61.2 (2007): 137-141.

12. Bai Y., et al. "Expression of human papillomavirus DNA in cholesteatoma of the middle ear". Zhonghua Er Bi Yan Hou Ke Za Zhi 35.5 (2000): 352-355.

13. Bergmann K., et al. "Human-papillomavirus DNA in cholesteatomas". International Journal of Cancer 59.4 (1994): 463466.

14. Ferekidis E., et al. "Correlation of clinical and surgical findings to histological features (koilocytosis, papillary hyperplasia) suggesting papillomavirus involvement in the pathogenesis of cholesteatoma". Medical Science Monitor 12.9 (2006): CR368-CR371.

15. Chao WY., et al. "Detection of human papillomavirus in cholesteatomas". European Archives of Oto-Rhino-Laryngology 257.3 (2000): 120-123.

16. Franz P., et al. "Prevalence of human papillomavirus DNA in cholesteatomas". ORL:Journal for Oto-Rhino-Laryngology and its Related Specialties 69.4 (2007): 251-255.

\section{Volume 1 Issue 4 April 2018}

(C) All rights are reserved by Charalampos Skoulakis., et al. 